Original Article

\title{
Using health games for rehabilitation of patients with infantile cerebral palsy
}

\author{
Wan-Chen Lee, Msc ${ }^{1)}$, Miriam C. Reyes-Fernández, Bsc ${ }^{1)}$, Rubén Posada-Gómez, PhD ${ }^{1}{ }^{*}$, \\ UlisesJuáReZ-Martínez, $\mathrm{PhD}^{1)}$, AlbinoMartíneZ-SibajA,PhD ${ }^{1)}$,GinerAlor-Hernández,PhD ${ }^{1)}$ \\ 1) Research Division and Postgraduate Studies, Instituto Tecnológico de Orizaba: \\ Av. Instituto Tecnológico 852, Col. Emiliano Zapata, Orizaba 94300, México
}

\begin{abstract}
Purpose] The purposes of this study were to evaluate whether the therapeutic games developed by the study team are significantly effective for upper limb rehabilitation of patients with cerebral palsy and to assess the development of the games and the evolution of patients throughout the therapy sessions. [Subjects and Methods] This study demonstrates the results of using therapeutic games in patients with infantile cerebral palsy. The therapies were performed in 30-minute sessions for about 1 to 4 months. This study shows the progress of five children with cerebral palsy during the sessions. The time it took the children on each road and the times required to complete a task were measured. In addition, the level of difficulty of the games was gradually increased at each session. [Results] Results have shown good progress on the accuracy of the movements and an increase in concentration level during the execution of the games, showing an improvement in the patients' performance by $40-55 \%$ faster. [Conclusions] Health games encourage children to comply with therapy. The advantage of the game is that the patient can perform the therapy at home, which could help achieve further progress in patients.

Key words: Infantile cerebral palsy, Rehabilitation games, Computer games
\end{abstract}

(This article was submitted Feb. 18, 2016, and was accepted May 14, 2016)

\section{INTRODUCTION}

Cerebral palsy (CP) is a no progressive disorder of the immature brain that results in movement and postural control impairments. CP is the most common cause of physical disability in childhood ${ }^{1)}$. The syndrome of CP encompasses a large group of childhood movement and postural disorders. The incidence of $\mathrm{CP}$, a lifelong disorder is 2-3.5 cases per 1,000 live births $^{2,3)}$ and has remained stable in the past 40 years. Therefore, studies and research on children with $\mathrm{CP}$ are important for the improvement of patients' disabilities.

Weightman et al. ${ }^{4)}$ showed that the use of computer games is good and provides a positive result for the recovery of muscle movements in children with CP. To help improve the movement of the upper limbs of children with CP, therapeutic games have been designed, especially for upper limb rehabilitation.

In recent years, the importance of using technology to help improve therapies and patient assessments has become accepted in rehabilitation. Human integration of a computer or robot has also been used in the medical field for rehabilitation ${ }^{5,6)}$. The use of video games to help patients with their rehabilitation therapy has shown positive results ${ }^{7}$, particularly the use of Nintendo $\mathrm{Wii}^{\odot}$ games in rehabilitation ${ }^{5,8-10)}$.

Videogame therapy has also been used in elderly patients, resulting in a significant improvement in balance and mobility in these patients in comparison with other participants who received traditional therapy in a conventional treatment room ${ }^{8,11)}$. Li et al. ${ }^{12)}$ showed that the video game exercise could engage participants" attention for a longer period than traditional and tracking exercises. Keith et al. ${ }^{13)}$ showed that integrating game into therapy can increase the time spent by patients in therapy. In addition, they suggest that through detailed research and development, video games might be a safe, cost-efficient, and

*Corresponding author. Rubén Posada-Gómez (E-mail: pgruben@yahoo.com)

(C)2016 The Society of Physical Therapy Science. Published by IPEC Inc.

This is an open-access article distributed under the terms of the Creative Commons Attribution Non-Commercial No Derivatives (by-nc-nd) License $<$ http://creativecommons.org/licenses/by-nc-nd/4.0/>. 
effective supplement to conventional physical therapy. After using Nintendo Wii ${ }^{\odot}$ games in rehabilitation, $75 \%$ of patients indicated subjectively that it seemed as useful as, or even better, than conventional rehabilitation, $81.3 \%$ found it fairly enjoyable, $87.5 \%$ wanted to continue using it as part of their rehabilitation program, and $93.8 \%$ were willing to recommend it to other patients ${ }^{5}$.

King et al. showed that the use of interactive platforms is more attractive for patients than performing tedious and re-

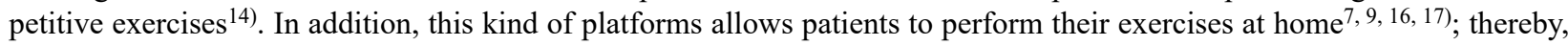
patients are able to increase their therapy time. The additional tools used in rehabilitations, such as computer programs, are easy to install and physically simple, light, and small. For all these advantages, the computer is a powerful support tool in rehabilitation. Likewise, the augmented reality has proved more effective than conventional treatments ${ }^{18)}$. However, neurorehabilitation usability studies have found that off-the-shelf games may actually provide negative additive and visual feedback because patients are not fast enough or efficient enough to perform the movements required to play the game successfully ${ }^{7)}$.

Shah et al. ${ }^{19)}$ reported that rehabilitation games need to be designed specifically for the purpose of rehabilitation and be customizable according to individual needs. Several studies have shown the benefits of using games developed specifically for rehabilitation purposes ${ }^{19,20)}$.

Hence, games specifically for rehabilitation of the upper limbs were developed, focusing on application in children with $\mathrm{CP}$, in order to achieve adequate support for patients. This project allows interaction with the physician, in which the physician can design the path of the game when a specific movement of the patient is required. A great advantage of this rehabilitation game is that it can be customized to suit the specific needs of individual patients.

\section{SUBJECTS AND METHODS}

In order to use this therapeutic game that the study team developed, a regular computer should be connected to a large screen, TV, or projector. A webcam or camera (EyeCamera PlayStation $3^{\odot}$ ) should be connected to the same computer to video-record the whole process during the game. Patients use a red, blue, or green LED pencil to install and manipulate the game (Fig. 1).

The reason for using a projector in the case study was that the size of the image was adjustable; therefore, adjustment could be made depending on the range of motion of the patient's arms. The LED pencil could be turned on in two ways as follows: switch to the top or button, or retain in the middle. Each patient's ability to move the fingers was considered. Another prototype bracelet LED was also made for the patients who could not hold a LED pencil. The LED was placed inside a small sphere of glass and filled with hot melt. When the pencil was switched on, the LED light was focused in different directions (Fig. 1). As LED is unidirectional, light is focused on the top. If the top LED switch is turned on in another direction, the light that seen on the camera will be markedly decreased or even disappears.

Two sets of rehabilitation games were developed to stimulate the upper limb, in which patients were required to perform flexion, extension, pronation, and supination of the arm movements and hand grip, which are the fine motor skills of the arms and hands. The rehabilitation game was designed with different levels of difficulty that can be played depending on the outcome in patients. There by, patients are encouraged to improve their fine mobility.

In order to perform this therapeutic game, before the game starts, the patient needs to hold the LED pen and point it to the car movement on the screen during the game. When the game starts, the patient moves the LED pen and follows the road to the final destination. The patient should maintain the car inside the road. When the patient pushes the car on the screen off the road, an error will be recorded. At the end of each level, information such as playing time, number of errors, and the patient's evolution will be recorded (Figs. 2 and 3).

The objective of the game is to stimulate patients to move their arms in straight, curved, circle, square, rectangle, spiral, and triangle lines, among other movements. At the most-advanced level, the roads are more complex and the widths of the roads are narrower (Table 1 and Fig.4). The more the patients can focus and perform more-complex and more-precise movements, the better are the results obtained.

The second game is called frog eating pastry. This game has a similar purpose to the previous one. It consists of a frog eating pastry while avoiding touching moving bombs (Fig. 2). In this game, the movements are not limited in order to capture all the pastry and finish the game. During the game, the patients have to move their arms in straight, curved, and rectangular lines, among other types of movement, but with more freedom and faster. This game focuses on agility of arm movements. The patients can exercise their thumbs while pressing the button at the same time.

The previous two games, besides being useful for exercising the arms, are also useful for children to identify colors and images, and differentiate functions. The differentiation is done by accounting and identifying the different sounds and their meanings as presented in the games. When the patient goes to wrong way, a beeping sound would signal for the error. When the game ends, an applause sound is made. When the patient fails to point the car to the correct direction, a sound will signal the patient to correct the error.

All the experiments were completed in accordance with the Ethical Principles for Medical Research Involving Human Subjects of the Declaration of Helsinki developed by the World Medical Association ${ }^{20)}$. Thereby, the risks for the participants in the performance of the tests were minimized as much as possible. In addition, this study was reviewed by the institutional board. All the participants were informed about the procedures of the test, and their written consent were obtained before the study. 


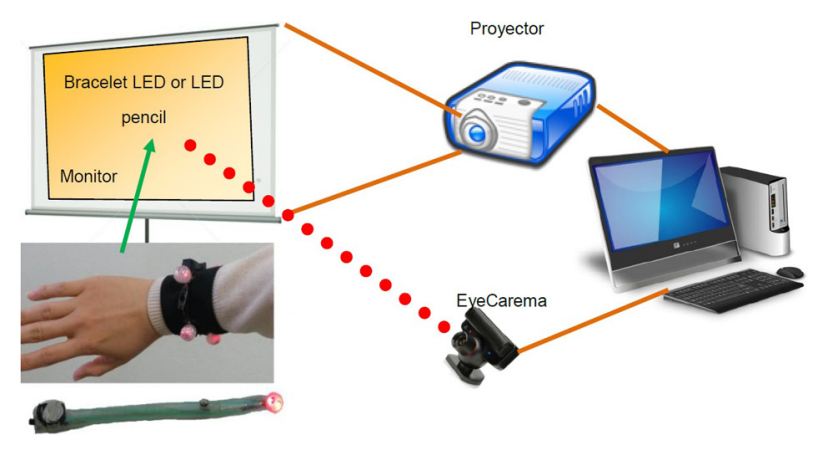

Fig. 1. Description map of installation using LED pencil or bracelet LED

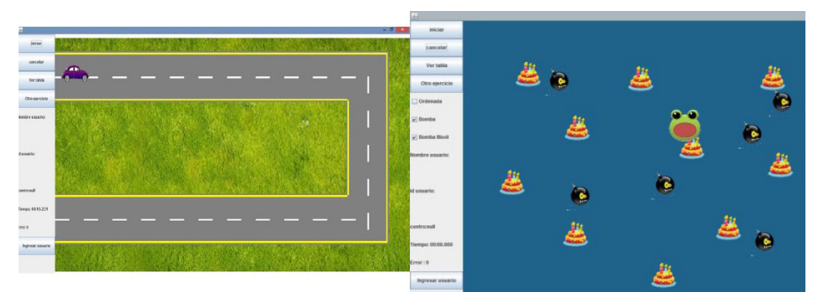

Fig. 2. Car game and frog game

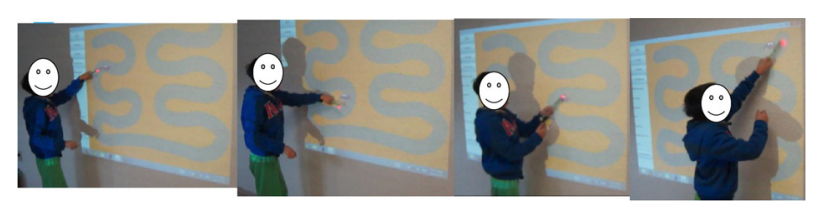

Fig. 3. Patient perfomed the game

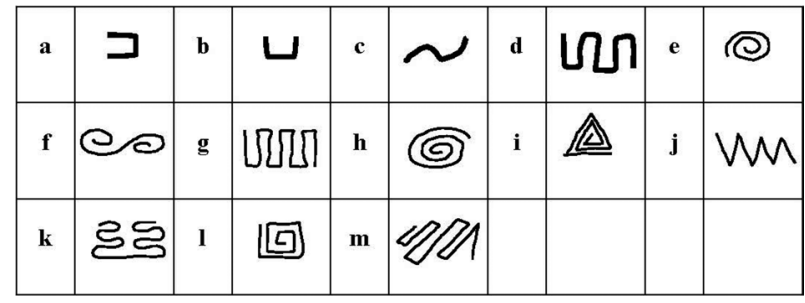

Fig. 4. Shaped road

Table 1. Road game

\begin{tabular}{lccc}
\hline Level & $\begin{array}{c}\text { Road width } \\
(\mathrm{cm})\end{array}$ & $\begin{array}{c}\text { Road length } \\
(\mathrm{cm})\end{array}$ & Shaped road \\
\hline Level 1 & 20 & 285 & Fig. 4-a \\
Level 1A & 20 & 230 & Fig. 4-b \\
Level 2 & 21 & 190 & Fig. 4-c \\
Level 3 & 16 & 490 & Fig. 4-d \\
Level 4 & 16 & 390 & Fig. 4-e \\
Level 4 A & 15 & 400 & Fig. 4-f \\
Level 5 A & 11 & 660 & Fig. 4-g \\
Level 5 B & 11 & 430 & Fig. 4-h \\
Level 6 A & 7 & 450 & Fig. 4-i \\
Level 6 B & 7 & 500 & Fig. 4-j \\
Level 6 C & 7 & 550 & Fig. 4-k \\
Level 7 A & 7 & 530 & Fig. 4-1 \\
Level 7 B & 7 & 600 & Fig. 4-m \\
\hline
\end{tabular}

\section{RESULTS}

In order to achieve the purpose of this study, children with CP aged between 6 and 12 years who had some difficulty of the upper limbs and hand movement but still able to perform the game were selected. This study was conducted with five children with $\mathrm{CP}$ whose ages ranged from 6 to 10 years. The duration of playing the game for its therapeutic purpose is between 30 and 135 days. Table 2 presents the data of the patients at baseline and after performing the game therapy.

The progress of the patients in this study was based on the level of the games and errors they made. The error refers to the number of times the patients deviated from the road between the first and last weeks. The duration of the exercise represents the degree of active movement of the arms and forearms. The number of times the patients went off the road indicates limitations of active movement, flexion and extension of the fingers, wrists, and forearms.

Table 3 presents the results of the average racing game that were played by patients for 16 weeks. It shows the average time in seconds and the number of times the patients got off a defined road in the first 2 weeks and last 2 weeks.

According to the results presented in Table 3, are makeable progress occurred between $\mathrm{T}_{0}$ and $\mathrm{T}_{1}$; the results demonstrated an error reduction rate from $40 \%$ to $55 \%$. The patients had improved their active movement, and flexion and extension of arms and forearms. At the beginning of the game, the patients found it difficult to raise and extend their arms. When they started exercising, their arms got tired easily. After several months, the patients were able to extend their arms a little more and did not get tired easily. Table 3 also shows improvement in terms of correctly drawing the car within the defined road. In the basic road, the speed was reduced by $49 \%$. However, the number of times the direction deviated from the road was not reduced. The standard deviation of the number times the direction deviated from the road in the basic level was 38.34 . It is because some patients wanted to achieve their target levels eagerly and did not pay much attention about how well they were able to finish the game.

In the medium level of the game, results show a 55\% increase in speed and $49 \%$ correct movements inside the road. The numbers demonstrate significant improvement in flexion and extension of the hands, wrist, and fingers. After the therapeutic 
Table 2. Data of the patients

\begin{tabular}{|c|c|c|c|c|c|}
\hline & $\mathrm{P} 1$ & $\mathrm{P} 2$ & $\mathrm{P} 3$ & $\mathrm{P} 4$ & P5 \\
\hline Age (yrs) & 10 & 9 & 8 & 6 & 7 \\
\hline Hand to exercise & Left & Right & Left & Right & Right \\
\hline Height (m) & 1.45 & 1.40 & 1.35 & 1.20 & 1.30 \\
\hline Weight (kg) & 38 & 40 & 30 & 32 & 35 \\
\hline Espasticity & $\begin{array}{l}\text { Left hand } \\
\text { and left foot }\end{array}$ & $\begin{array}{c}\text { Feet, back, arms, } \\
\text { and hands }\end{array}$ & $\begin{array}{l}\text { Feet, back, arms, } \\
\text { and hands }\end{array}$ & Hands and feet & Hands and feet \\
\hline $\begin{array}{l}\text { Exercise time } \\
\text { (months) }\end{array}$ & 4.5 & 1.5 & 2 & 1 & 2 \\
\hline $\begin{array}{l}\text { Session duration } \\
\text { (min) }\end{array}$ & 30 & 30 & 30 & 30 & 30 \\
\hline Position & Stand & Sit & Sit & Stand & Stand \\
\hline $\begin{array}{l}\text { Hand commonly } \\
\text { used }\end{array}$ & Right & Right & Left & Right & Right \\
\hline $\begin{array}{l}\text { Initial } \\
\text { description }\end{array}$ & $\begin{array}{c}\text { Can walk, write } \\
\text { with the right hand, } \\
\text { but weak left hand }\end{array}$ & $\begin{array}{l}\text { Cannot stand up, cannot } \\
\text { straighten the back, cannot } \\
\text { take with both hands, and } \\
\text { have difficulty speaking }\end{array}$ & $\begin{array}{l}\text { Cannot stand up, cannot } \\
\text { straighten the back, cannot } \\
\text { take with both hands, and } \\
\text { have difficulty speaking }\end{array}$ & $\begin{array}{l}\text { Does not have } \\
\text { good hand } \\
\text { movement }\end{array}$ & $\begin{array}{l}\text { Does not have } \\
\text { good hand } \\
\text { movement }\end{array}$ \\
\hline $\begin{array}{l}\text { GMFCS } \\
\text { level }\end{array}$ & I & IV & IV & II & II \\
\hline
\end{tabular}

Table 3. Results of the average racing game

\begin{tabular}{lcccc}
\hline & T0 & T1 & $\begin{array}{c}\text { \%reduced } \\
\text { time }\end{array}$ & SD* \\
\hline Speed taken to finish a basic-level game (levels 1-4), s & 41 & 24.4 & $40 \%$ & 11 \\
Speed taken to finish a medium-level game (level 4A-5B), s & 78 & 35.2 & $55 \%$ & 7.1 \\
Speed taken to finish an advanced-level game (levels 6 or7), * s & 63.1 & 34 & $46 \%$ & ---- \\
Number of times the patients got off a defined road: width, 20-16 cm (levels 1-4) & 5.24 & 5.26 & $-6 \%$ & 38.34 \\
Number of times the patients got off a defined road: width, 15-11 cm (levels 4A-5B) & 18.7 & 9.5 & $49 \%$ & 14.27 \\
Number of times the patients got off a defined road: width, 7 cm (level 6 or 7)* & 14.3 & 8.46 & $41 \%$ & ---- \\
\hline
\end{tabular}

${ }^{*}$ Level achieved only by patient 1 (P1), T0: Average result of the first 2 weeks, T1: Average result of the last 2 weeks, SD: Standard deviation of the percentage of time reduction for all the patients

games, the patients could hold the marker better, displaying better agility of the finger to press the button to start the game and better wrist movements. These improvements were caused by the reduction in the number of times the direction deviated from the road. However, at the advanced level, only one patient (P1) achieved the goal. The game is designed to allow the patient to enter the next level after completing the previous levels.

The standard deviation of the increases in speed of the five patients was 11 and 7.1. The results showed a similar increase in speed among the patients. However, among those patients, patient 1 participated the longest time ( 4.5 months) in the study. He also had better study results, $63.7 \%$ increase in speed in the medium level, and an average of $74 \%$ reduction in the number of times he deviated from a defined road.

Even though the therapeutic duration of each patient varied, the improvement of each patient was noticeable. The patients who participated in the game longer showed significant improvements, including the reduction in the time to finish the game, better movement inside the defined road, and better ability to hold the marker.

At the end of the therapies, the participants were interviewed for their impression about the games. Several questions were asked, such as the following: "Do you like this therapeutic game?" "Do you find the games interesting to use?" "Would you like to continue performing this game?" The survey results show that the participants felt interested to use the rehabilitation games, and the duration of the therapies was increased. The participants paid more attention to the therapy and enjoyed the games.

Table 4 shows the comparison of the activities and movement of the patients between the baseline and after the game rehabilitation therapy. 
Table 4. Comparison between before and after the experiment

\begin{tabular}{|c|c|c|}
\hline & Before the experiment & After the experiment \\
\hline $\mathrm{P} 1$ & Left hand, wrist, and fingers weak & $\begin{array}{l}\text { Left hand had enough strength to catch a ball and turn a key to } \\
\text { open the door, among other fine motor exercises }\end{array}$ \\
\hline $\mathrm{P} 2$ & Could not take with both hands & $\begin{array}{l}\text { Could paste paper inside a shape and draw a straight line on } \\
\text { paper, could hold the pencil harder, could paint hard and inside a } \\
\text { picture frame, and could cut sheets of paper straighter than before }\end{array}$ \\
\hline P3 & $\begin{array}{l}\text { Could not hold well the marker and had trouble moving } \\
\text { the arm and point maker inside the game route }\end{array}$ & Could hold the marker better and had better arm movement \\
\hline $\mathrm{P} 4$ & $\begin{array}{l}\text { Could not hold well the marker and had trouble with } \\
\text { wrist movement }\end{array}$ & Had better arm and wrist movements \\
\hline P5 & $\begin{array}{l}\text { Could not hold well the marker and had trouble moving } \\
\text { the arm and point maker inside the game route }\end{array}$ & Could hold the marker better and had better arm movement \\
\hline
\end{tabular}

\section{DISCUSSION}

The study results from the five patients provide a breakthrough in the movement abilities of the arms and hands of the patient. These results show that rehabilitation games can be a good tool to support therapy for children with CP. Such treatment not only focuses on arm movement but also helps identify images, colors, and sounds. This treatment can also train arm movements within specific details. These games can encourage children to exercise because they become more motivated to perform the therapies ${ }^{12-14}$. In addition, another advantage is that patients can perform exercises at home with less restrictions ${ }^{7,9,15,16)}$.

In the present study, the comparison between before and after the experiment showed significant improvement in the speed and fine movements of the children. Only five patients were selected in the present study. However, more patients are required to obtain more reliable results. The main contribution of this research is to demonstrate that therapeutic games can provide additional help for patients with fine-movement problems in their arms and hands. Moreover, the devices used in these games are economical and flexible.

In future studies, the number of cases should be increased to ensure the validity of study results. Patients are able to play the rehabilitation game at home and thus achieve faster improvement. In addition, other studies on other types of disabilities should be conducted.

\section{REFERENCES}

1) Kawakami M, Liu M, Otsuka T, et al.: Asymmetric skull deformity in children with cerebral palsy: frequency and correlation with postural abnormalities and deformities. J Rehabil Med, 2013, 45: 149-153. [Medline] [CrossRef]

2) Drużbicki M, Rusek W, Snela S, et al.: Functional effects of robotic-assisted locomotor treadmill thearapy in children with cerebral palsy. J Rehabil Med, 2013, 45: 358-363. [Medline] [CrossRef]

3) Jones MW, Morgan E, Shelton JE: Primary care of the child with cerebral palsy: a review of systems (part II). J Pediatr Health Care, 2007, 21: 226-237. [Medline] [CrossRef]

4) Weightman A, Preston N, Levesley M, et al.: Home based computer-assisted upper limb exercise for young children with cerebral palsy: a feasibility study investigating impact on motor control and functional outcome. J Rehabil Med, 2011, 43: 359-363. [Medline] [CrossRef]

5) Yong Joo L, Soon Yin T, Xu D, et al.: A feasibility study using interactive commercial off-the-shelf computer gaming in upper limb rehabilitation in patients after stroke. J Rehabil Med, 2010, 42: 437-441. [Medline] [CrossRef]

6) Patel S, Park H, Bonato P, et al.: A review of wearable sensors and systems with application in rehabilitation. J Neuroeng Rehabil, 2012, 9: 21. [Medline] [CrossRef]

7) Radtka S, Hone R, Brown C, et al.: Feasibility of computer-based videogame therapy for children with cerebral palsy. Games Health J, 2013, 2: 222-228. [Medline] [CrossRef]

8) Laver K, George S, Ratcliffe J, et al.: Use of an interactive video gaming program compared with conventional physiotherapy for hospitalised older adults: a feasibility trial. Disabil Rehabil, 2012, 34: 1802-1808. [Medline] [CrossRef]

9) Peters DM, McPherson AK, Fletcher B, et al.: Counting repetitions: an observational study of video game play in people with chronic poststroke hemiparesis. J Neurol Phys Ther, 2013, 37: 105-111. [Medline] [CrossRef]

10) Tarakci D, Ozdincler AR, Tarakci E, et al.: Wii-base balance therapy to improve balance function of children with cerebral palsy: a pilot study. J Phys Ther Sci, 2013, 25: 1123-1127. [Medline] [CrossRef]

11) Morone G, Tramontano M, Losa M, et al.: The efficacy of balance training with video game-based therapy in subacute stroke patients: a randomized controlled trial. Biomed Res Int, 2014. 
12) Li C, Rusák Z, Horváth I, et al.: Influence of complementing a robotic upper limb rehabilitation system with video games on the engagement of the participants: a study focusing on muscle activities. Int J Rehabil Res, 2014, 37: 334-342. [Medline] [CrossRef]

13) Lohse K, Shirzad N, Verster A, et al.: Video games and rehabilitation: using design principles to enhance engagement in physical therapy. J Neurol Phys Ther, 2013, 37: 166-175. [Medline] [CrossRef]

14) King M, Hijmans J, Sampson M, et al.: Home-based stroke rehabilitation using computer gaming. NZ J Physio-ther, 2012, 40: 128-134.

15) Lange BS, Flynn SM, Rizzo AA: Initial usability assessment of the off-the-shelf video game consoles for clinical game-based motor rehabilitation. Phys Ther Rev, 2009, 14: 355-363. [CrossRef]

16) Lange B, Flynn S, Proffitt R, et al.: Development of an interactive game-based rehabilitation tool for dynamic balance training. Top Stroke Rehabil, 2010, 17: 345-352. [Medline] [CrossRef]

17) Laver K, George S, Ratcliffe J, et al.: Virtual reality stroke rehabilitation—hype or hope? Aust Occup Ther J, 2011, 58: 215-219. [Medline] [CrossRef]

18) Shah N, Basteris A, Amirabdollahian F: Design parameters in multimodal games for rehabilitation. Games Health J, 2014, 3: 13-20. [Medline] [CrossRef]

19) Shapi’i A, Mat Zin NA, Elaklouk AM: A game system for cognitive rehabilitation. BioMed Res Int, 2015.

20) Korean Medical Association: World Medical Association Declaration of Helsinki: ethical principles for medical research involving human subjects. J Korean Med Assoc, 2014, 57: 899-902 [CrossRef]. 